\title{
SIGNA. REVISTA DE LA ASOCIACIÓN ESPAÑOLA DE SEMIÓTICA
}

\author{
Alicia Yllera \\ Universidad Nacional de Educación a Distancia
}

Desde que, en noviembre de 1986, fui elegida Presidenta de la Asociación Española de Semiótica, en su II Congreso celebrado en la Universidad de Oviedo, había proyectado la creación de una revista de la Asociación. Además de la publicación de las actas de los Coloquios, celebrados cada dos años, la Asociación contaría así con un órgano de expresión anual. Las dificultades financieras impidieron, durante un tiempo, que el proyecto se concretase. Sólo en 1992, gracias a la ayuda de la Universidad Nacional de Educación a Distancia (UNED, Madrid), pudo aparecer la revista. Desde esta fecha se han publicado siete volúmenes. El ritmo de publicación es de un volumen por año. El director se renueva cada cuatro años y forman parte de su comité de redacción tanto el Presidente como el Secretario de la Asociación. Mi sucesor en la dirección de la revista es José Romera Castillo y el secretario actual, Mario García-Page. La UNED es responsable de su publicación, distribución y suscripción (c/ Bravo Murillo, 38, 28015 MADRID). 
La revista consta, en general, de tres partes que comprenden, respectivamente, los artículos, el estado presente de los estudios sobre un tema dado y las reseñas de obras.

El Comité de Redacción intenta alternar los números de tema libre con los números en gran parte dedicados a un tema monografico. Los números 1 (1992), 4 (1995), 5 (1996) y 7 (1998) son en buena medida monográficos. El número 1 (1992) se dedicó a Ch. S. Peirce. Contiene las Actas de las sesiones plenarias y de las comunicacioens presentadas al I Seminario Internacional de Literatura y Semiótica, celebrado en la sede regional de la UNED en Segovia, los días 3-5 de julio de 1991. Comprende cuatro sesiones plenarias, dos de ellas dedicadas al análisis de las posibilidades de aplicar las teorías peircianas a la literatura (comunicaciones presentadas por Dinda L. Gorlée y Lucia Santaella Braga), una tercera versa sobre la semiosis (Floyd Merrell) y la cuarta es un trabajo de Robert Marty sobre los niveles y las pasarelas en semiótica textual. Otras Siete comunicaciones completan el número; algunas consisten en una aplicación de los principios peircianos a textos literarios, otras debaten ciertas cuestiones teóricas o analizan las relaciones entre Peirce y otros autores. El volumen se cierra con tres estados de la cuestión: el primero sobre el tema de Peirce y la literatura (Lucia Santaella Braga), el segundo sobre Peirce en España (Wenceslao Castañares) y el tercero a la vez sobre Peirce en España y España en Peirce (Jaime Nubiola).

El número 4 (1995) está en parte consagrado a Iuri M. Lotman. Incluye la traducción española de uno de sus textos publicados en ruso en 1992. El estudio versa sobre la biografía literaria y se analiza en él la formación de las normas de la biografía de escritor en la literatura rusa a partir del siglo XVIII. Sigue un trabajo de su hijo, Mijaíl I. Lotman, que examina las bases filosóficas de la semiótica de la escuela de TartuMoscú y resalta la influencia de Kant sobre Lotman, mientras que fue Hegel el que mayor impronta dejó sobre la filosofía rusa. Un tercer texto de un miembro de la escuela de Tartu, Peeter Torop, compara las dificultades metalinguíísticas de la teoría de la traducción con las de la teoría semiótica. Manuel Cáceres Sánchez (de la Universidad de Granada) completa estos artículos con una bibliografía sobre Lotman, en español, francés, inglés, italiano, portugués y alemán, que es una puesta al día de la que había publicado previamente en la revista Discurso, en $1993{ }^{1}$.

1 Publica la revista Discurso la Asociación Semiótica Andaluza. La Asociación Semiótica Vasca publicó en 1991 un primer número de su revista Era que, hasta el presente, no ha tenido continuación. 
El número 5 (1996) comprende un homenaje a Emmanuel Lévinas y un cierto número de artículos de tema libre. Los trabajos de Augusto Ponzio (Universidad de Bari, Italia), Miguel García-Baró (Universidad Complutense de Madrid), César Moreno Márquez (Universidad de Sevilla), Diego Sánchez Meca (UNED) y Antonio Domínguez Rey (UNED) analizan la obra y el pensamiento del autor citado.

El número 6 (1997) carece del carácter monográfico de los anteriormente reseñados, pese a que tres artículos tratan de la Generación del 27: uno de ellos estudia a un crítico literario, José Fernández Montesinos (Francisco Abad), otro a un gramático, Salvador Fernández Ramírez (del que Bienvenido Palomo Olmos, de la Universidad Autónoma de Madrid, presenta una bibliografía comentada) y el último a un poeta, Pedro Salinas, cuya lengua poética analiza M. ${ }^{\mathrm{a}}$ Luisa Peces.

El número 7 (1998) consta de una primera parte en la que se traza un panorama de la semiótica en el ámbito hispánico, panorama que se completará en un próximo número con presentaciones de la semiótica en Argentina, Colombia, Cuba, Perú y España. En este número se analiza la actividad de la Federación Latinoamericana de Semiótica desde su fundación (Lucrecia Escudero) y se presenta un panorama de la semiótica en Chile (Rafael del Villar Muñoz), México (Adrián S.Gimate-Welsh), Puerto Rico (Eliseo R. Colón), Venezuela (José Enrique Finol y Dobrila Djukich) y Uruguay (Marisol Álvarez y Richard Danta; Claudia González Costanzo).

La diversidad caracteriza, como era de esperar, a los artículos de tema libre. Componen la mayor parte de los números 2 (1993), 3 (1994) y 6 (1997). Los trabajos de semiótica literaria, incluso de teoría literaria, son los más numerosos, lo que no puede sorprender puesto que la publicación de la revista corre a cargo de la Facultad de Filología de la UNED. Los estudios de semiótica lingüística, de semiótica de la imagen, de semiótica de los medias, etc. son menos numerosos. Sin embargo, el comité de redacción es bien consciente de la necesidad de ampliar los temas tratados. En los últimos números, figuran un cierto número de trabajos sobre el cine así como algunas contribuciones sobre semiótica de la imagen o de la prensa, lo que presagia una apertura creciente a otros dominios no literarios. Algunos estudios atienden a las relaciones entre las artes, el cine y la literatura o la literatura en la televisión. Otros analizan los graffiti o figuras retóricas como el retruécano léxico. En el dominio de la crítica literaria, diversos artículos estudian la crítica feminista, las teorías peircianas, bajtinianas, barthesianas, ricoeurianas, deleuzianas, kristevianas, oulipianas, derri- 
dianas, etc. Otros versan sobre cuestiones de géneros literarios o de narratología, pues los estudios aplicados a textos narrativos son más numerosos que los estudios que atañen a obras líricas o dramáticas. La mayoría de estos estudios de semiótica literaria se interesan por obras escritas en lengua española, por estudiosos españoles o hispanomericanos. Son menos numerosos los trabajos sobre otras literaturas.

Signa ha logrado superar los siete números consecutivos, lo que parece augurarle un buen porvenir. Únicamente desearíamos darle una mayor diversidad y ampliar sus temas de investigación.

La mayoría de los colaboradores son, hoy por hoy, investigadores españoles, seguidos a distancia por los estudiosos latinoamericanos y finalmente los europeos. La revista está, sin embargo, abierta a investigadores de todos los países, y desearíamos que esta breve reseña fuese una invitación a colaborar con nosotros. 